\title{
Radioulnar synostosis-developmental delay-hypotonia syndrome
}

INSERM

\section{Source}

INSERM. (1999). Orphanet: an online rare disease and orphan drug data base. Radioulnar synostosis-developmental delay-hypotonia syndrome. ORPHA:3270

Radioulnar synostosis-developmental delay-hypotonia syndrome, also known as Der Kaloustian-McIntosh-Silver syndrome, is an extremely rare syndrome with synostosis described in about 4 patients to date with clinical manifestations including congenital unilateral radioulnar synostosis, generalized hypotonia, developmental delay, and dysmorphic facial features (long face, prominent nose and ears). 